\title{
Convergence study of a DDFV scheme for the Navier-Stokes equations arising in the domain decomposition setting
}

Thierry Goudon, Stella Krell and Giulia Lissoni

\begin{abstract}
We consider DDFV discretization of the Navier-Stokes equations where the convection fluxes are computed by means of $B$-schemes, generalizing the classical centered and upwind discretizations. This study is motivated by the analysis of domain decomposition approaches. We investigate on numerical grounds the convergence of the method.
\end{abstract}

MSC2010: 65M08, 76D05, 35Q35.

\section{Introduction}

We consider the incompressible Navier-Stokes problem

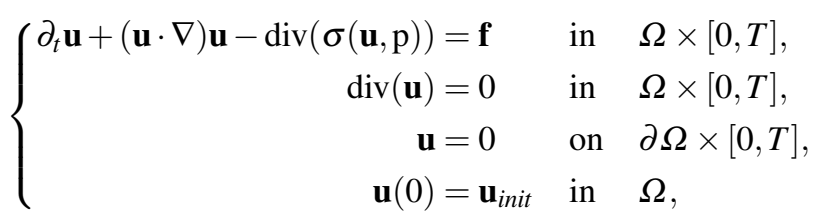

where $\Omega$ is an open connected bounded polygonal domain of $\mathbb{R}^{2}, \mathbf{f} \in\left(L^{2}(\Omega)\right)^{2}$ and $\mathbf{u}_{\text {init }} \in\left(L^{\infty}(\Omega)\right)^{2}$ given. The unknowns $\mathbf{u}: \Omega \times[0, T] \rightarrow \mathbb{R}^{2}$ and $\mathrm{p}: \Omega \times[0, T] \rightarrow \mathbb{R}$ are respectively the velocity and the pressure; $\sigma(\mathbf{u}, \mathrm{p})=\frac{2}{\mathrm{Re}} \mathrm{Du}-$ pId stands for the stress tensor, and $\operatorname{Re}>0$ is the Reynolds number. Here and below, the strain rate tensor is defined by the symmetric part of the velocity gradient $D \mathbf{u}=\frac{1}{2}\left(\nabla \mathbf{u}+{ }^{t} \nabla \mathbf{u}\right)$.

Thierry Goudon e-mail: thierry.goudon@inria.fr Stella Krell e-mail: stella.krell@univ-cotedazur.fr Giulia Lissoni*e-mail: giulia.lissoni@univ-nantes.fr

Université Côte dAzur, CNRS, Inria, LJAD, France

*Université de Nantes, LMJL, CNRS 
The Discrete Duality Finite Volume (DDFV) approach is quite appealing because it applies to very general meshes and it mimics at the discrete level the dual properties of the continuous differential operators. The introduction of the DDFV formalism dates back to $[3,5,9]$, in order to approximate anisotropic diffusion problems on general meshes, including non-conformal and distorted meshes. DDFV schemes require unknowns on both the vertices and centers of primal control volumes; in particular, for the Stokes and Navier-Stokes problems it leads naturally to staggered discretizations of velocity and pressure; see $[1,4,6,10]$. This work is motivated by the analysis of DDFV domain decomposition methods for (1). In contrast to direct methods, domain decomposition methods, in which the computational domain is decomposed into smaller subdomains, are naturally parallel; this makes those methods interesting for high performance computing perspectives. The classical Schwarz algorithm was proposed in 1870 by H. A. Schwarz for the Laplace problem and further studied in 1990 by P.-L. Lions, see [12, 13]. This approach has been adapted to many problems and motivates a huge literature.

In [7], we investigated non overlapping Schwarz algorithms in the DDFV framework for the Navier-Stokes system. The convergence analysis of the Schwarz iterations reveals a complex interplay between the design of the transmission conditions and the definition of the numerical fluxes. It turns out that the discrete limit problem does not coincide with the "standard" DDFV scheme on the entire domain; instead fluxes near the interface need to be modified. We are going to show, based on numerical experiments, that the modified scheme still provides a good approximation of the solution of (1) on $\Omega$. Note that it is also possible to modify the fluxes of the domain decomposition method in order to restore a given DDFV scheme on $\Omega$. These considerations rely on the formalism on $B$-schemes $[2,8]$ which allows us to consider general convection fluxes.

\section{The DDFV framework}

We consider a domain $\Omega$ that can be seen as the union of two subdomains that share a common interface denoted by $\Gamma$.

Meshes: The complete description of the DDFV scheme for the 2D NavierStokes problem can be found in $[6,11]$. A DDFV mesh is a pair $(\mathfrak{T}, \mathfrak{D})$; $\mathfrak{T}$ combines the primal mesh $\mathfrak{M} \cup \partial \mathfrak{M}$ (whose cells are denoted by k), and the dual mesh $\mathfrak{M}^{*} \cup$ $\partial \mathfrak{M}^{*}$, (whose cells $\mathrm{K}^{*}$ are built around the vertices $x_{\mathrm{K}^{*}}$ of the primal mesh).

The primal mesh $\mathfrak{M}$ consists of disjoint polygons к called "primal cells", whose union covers $\Omega$. The symbol $\partial \mathfrak{M}$ denotes the set of edges of primal mesh included in $\partial \Omega$, that are considered as degenerated primal cells. We associate to each $\mathrm{k} \mathrm{a}$ point $x_{\mathrm{K}}$, called "center". For the cells of the boundary, the point $x_{\mathrm{K}}$ is situated at the middle point of the edge. For all the neighbors volumes $\mathrm{k}$ and $\mathrm{L}$, we suppose that $\partial \mathrm{k} \cap \partial_{\mathrm{L}}$ is a segment that we call $\sigma=\mathrm{k} \mid \mathrm{L}$, edge of the primal mesh $\mathfrak{M}$.

From this primal mesh, we build the associated dual mesh. A dual cell $\mathrm{K}^{*}$ is associated to a vertex $x_{\mathrm{K}^{*}}$ of the primal mesh. The dual cells are obtained by joining the centers of the primal cells that have $x_{\mathrm{K}^{*}}$ as vertex. Then, the point $x_{\mathrm{K}^{*}}$ is called 
center of $\mathrm{K}^{*}$. We will distinguish interior dual mesh, for which $x_{\mathrm{K}^{*}}$ does not belong to $\partial \Omega$, denoted by $\mathfrak{M}^{*}$ and the boundary dual mesh, for which $x_{\mathrm{K}^{*}}$ belongs to $\partial \Omega$, denoted by $\partial \mathfrak{M}^{*}$. We denote with $\sigma^{*}=\mathrm{K}^{*} \mathrm{~L}^{*}$ the edges of the dual mesh.

Next, $\mathfrak{D}$ stands for the diamond mesh, whose cells $\mathrm{D}=\mathrm{D}_{\sigma, \sigma^{*}}$ are built such that their principal diagonals are a primal edge $\sigma$ and a dual edge $\sigma^{*}$. Thus a diamond is a quadrilateral with vertices $x_{\mathrm{K}}, x_{\mathrm{L}}, x_{\mathrm{K}^{*}}$ and $x_{\mathrm{L}^{*}}$. Note that we have $\Omega=\bigcup_{D \in \mathfrak{D}} \mathfrak{D}$. We distinguish the diamonds that intersect the interface $\Gamma$ as $\mathfrak{D}^{\Gamma}=\left\{D_{\sigma, \sigma^{*}} \in\right.$ $\mathfrak{D}$, such that $\sigma \subset \Gamma\}$.

For a diamond cell D we note by $m_{\mathrm{D}}$ its measure, $m_{\sigma}$ the length of the primal edge $\sigma, m_{\sigma^{*}}$ the length of the dual edge $\sigma^{*}, \mathbf{n}_{\sigma K}$ the unit vector normal to $\sigma$ oriented from $x_{\mathrm{K}}$ to $x_{\mathrm{L}}, \mathbf{n}_{\sigma^{*} \mathrm{~K}^{*}}$ the unit vector normal to $\sigma^{*}$ oriented from $x_{\mathrm{K}^{*}}$ to $x_{\mathrm{L}^{*}}$. We denote also its sides by $\mathfrak{s}$ and their measure by $m_{\mathfrak{s}}$; see Fig. 1 for an illustration.

Finally, we denote by $\mathbf{f}_{\mathrm{K}}\left(\right.$ resp. $\left.\mathbf{f}_{\mathrm{K}^{*}}\right)$ the mean-value of the source term $\mathbf{f}$ on $\mathrm{k} \in \mathfrak{M}$ (resp. on $\mathrm{K}^{*} \in \mathfrak{M}^{*} \cup \partial \mathfrak{M}^{*}$ ).

Unknowns: The DDFV method for Navier-Stokes problem uses staggered unknowns. We associate to every к $\in \mathfrak{M} \cup \partial \mathfrak{M}$ an unknown $\mathbf{u}_{\mathrm{K}} \in \mathbb{R}^{2}$, to every $\mathrm{K}^{*} \in \mathfrak{M}^{*} \cup \partial \mathfrak{M}^{*}$ an unknown $\mathbf{u}_{\mathrm{K}^{*}} \in \mathbb{R}^{2}$ for the velocity and to every $\mathrm{D} \in \mathfrak{D}$ an unknown $\mathrm{p}^{\mathrm{D}} \in \mathbb{R}$ for the pressure. Those unknowns are collected in the families:

$\mathbf{u}_{\mathfrak{T}}=\left(\left(\mathbf{u}_{\mathrm{K}}\right)_{\mathrm{K} \in(\mathfrak{M} \cup \supset \mathfrak{M})},\left(\mathbf{u}_{\mathrm{K}^{*}}\right)_{\mathrm{K}^{*} \in\left(\mathfrak{M}^{*} \cup \partial \mathfrak{M}^{*}\right)}\right) \in\left(\mathbb{R}^{2}\right)^{\mathfrak{T}} \quad$ and $\quad \mathrm{p}_{\mathfrak{D}}=\left(\left(\mathrm{p}^{\mathrm{D}}\right)_{\mathfrak{D} \in \mathfrak{D}}\right) \in \mathbb{R}^{\mathfrak{D}}$.

We define the subspace of $\left(\mathbb{R}^{2}\right)^{\mathfrak{T}}$ that takes into account Dirichlet boundary conditions:

$$
\mathbb{E}_{0}=\left\{\mathbf{u}_{\mathfrak{T}} \in\left(\mathbb{R}^{2}\right)^{\mathfrak{T}} \text {, s. t. } \forall \mathrm{K} \in \partial \mathfrak{M}, \mathbf{u}_{\mathrm{K}}=0 \text { and } \forall \mathrm{K}^{*} \in \partial \mathfrak{M}^{*}, \mathbf{u}_{\mathrm{K}^{*}}=0\right\} .
$$

For $\mathbf{v} \in\left(H^{2}(\Omega)\right)^{2}$, we set $\mathbb{P}_{c}^{\mathfrak{T}}(\mathbf{v})=\left(\left(\mathbf{v}\left(x_{\mathrm{K}}\right)\right)_{\mathrm{K} \in \mathfrak{M} \cup \partial \mathfrak{M}},\left(\mathbf{v}\left(x_{\mathrm{K}^{*}}\right)\right)_{\mathrm{K}^{*} \in \mathfrak{M}^{*} \cup \partial \mathfrak{M}^{*}}\right)$.

Discrete operators: We define a piecewise constant approximation of the gradient operator denoted by $\nabla^{\mathfrak{D}}:\left(\mathbb{R}^{2}\right)^{\mathfrak{T}} \rightarrow\left(\mathrm{M}_{2}(\mathbb{R})\right)^{\mathfrak{D}}$,

$$
\nabla^{\mathrm{D}} \mathbf{u}_{\mathfrak{I}}:=\frac{1}{2 m_{\mathrm{D}}}\left[m_{\sigma}\left(\mathbf{u}_{\mathrm{L}}-\mathbf{u}_{\mathrm{K}}\right) \otimes \mathbf{n}_{\sigma \mathrm{K}}+m_{\sigma^{*}}\left(\mathbf{u}_{\mathrm{L}^{*}}-\mathbf{u}_{\mathrm{K}^{*}}\right) \otimes \mathbf{n}_{\sigma^{*} \mathrm{~K}^{*}}\right], \quad \forall \mathrm{D} \in \mathfrak{D} .
$$

To work with the Navier-Stokes problem, we also need to define the discrete strain rate tensor $\mathrm{D}^{\mathcal{D}}: \mathbf{u}_{\mathfrak{T}} \in\left(\mathbb{R}^{2}\right)^{\mathfrak{T}} \mapsto\left(\mathrm{D}^{\mathrm{D}} \mathbf{u}_{\mathfrak{T}}\right)_{\mathrm{D} \in \mathfrak{D}} \in\left(\mathrm{M}_{2}(\mathbb{R})\right)^{\mathcal{D}}$, such that:

$$
\mathrm{D}^{\mathrm{D}} \mathbf{u}_{\mathfrak{\tau}}=\frac{\nabla^{\mathrm{D}} \mathbf{u}_{\mathfrak{z}}+{ }^{t}\left(\nabla^{\mathrm{D}} \mathbf{u}_{\mathfrak{z}}\right)}{2}, \quad \text { for } \mathrm{D} \in \mathfrak{D},
$$

the discrete stress tensor $\sigma^{\mathfrak{D}}:\left(\mathbf{u}_{\mathfrak{x}}, \mathrm{p}_{\mathfrak{D}}\right) \in\left(\mathbb{R}^{2}\right)^{\mathfrak{T}} \times \mathbb{R}^{\mathfrak{D}} \mapsto\left(\sigma^{\mathbb{D}}\left(\mathbf{u}_{\mathfrak{z}}, \mathrm{p}_{\mathfrak{D}}\right)\right)_{\mathbb{D} \in \mathfrak{D}} \in$ $\left(\mathrm{M}_{2}(\mathbb{R})\right)^{\mathfrak{D}}$

$$
\sigma^{\mathrm{D}}\left(\mathbf{u}_{\mathfrak{T}}, \mathrm{p}_{\mathfrak{D}}\right)=-\left(\frac{2}{\operatorname{Re}} \mathrm{D}^{\mathrm{D}} \mathbf{u}_{\mathfrak{T}}-\mathrm{p}^{\mathrm{D}} \mathrm{Id}\right), \quad \text { for } \mathrm{D} \in \mathfrak{D},
$$

and the discrete divergence of a vector field of $\left(\mathbb{R}^{2}\right)^{\mathfrak{T}}$ as $\operatorname{div}^{\mathfrak{D}}: \mathbf{u}_{\mathfrak{I}} \in\left(\mathbb{R}^{2}\right)^{\mathfrak{T}} \mapsto$ $\left(\operatorname{div}^{\mathrm{D}} \mathbf{u}_{\mathfrak{T}}\right)_{\mathbb{D} \in \mathfrak{D}} \in \mathbb{R}^{\mathcal{D}}$ with $\operatorname{div}^{\mathrm{D}} \mathbf{u}_{\mathfrak{T}}=\operatorname{Tr}\left(\nabla^{\mathrm{D}} \mathbf{u}_{\mathfrak{T}}\right)$ for any $\mathrm{D} \in \mathfrak{D}$. 
To treat convection terms, it is convenient to define the scalar velocity fluxes $F_{\sigma \mathrm{K}}$ and $F_{\sigma^{*} \mathrm{~K}^{*}}$; their definition comes from [11], up to the boundary terms. They are approximations of the fluxes: $\int_{\sigma}\left(\mathbf{u} \cdot \mathbf{n}_{\sigma \mathrm{K}}\right) \rightsquigarrow F_{\sigma \mathrm{K}}\left(\mathbf{u}_{\mathfrak{T}}\right)$ and $\int_{\sigma^{*}}\left(\mathbf{u} \cdot \mathbf{n}_{\sigma^{*} K^{*}}\right) \rightsquigarrow F_{\sigma^{*} K^{*}}\left(\mathbf{u}_{\mathfrak{T}}\right)$. By defining $m_{\mathfrak{s}} G_{\mathfrak{s}, \mathrm{D}}=m_{\mathfrak{s}} \frac{\mathbf{u}_{\mathrm{K}}+\mathbf{u}_{K^{*}}}{2} \cdot \mathbf{n}_{\mathfrak{s} \mathrm{D}}$, for the primal edges, we impose:

$$
m_{\sigma} F_{\sigma \mathrm{K}}=-\sum_{\mathfrak{s} \in \partial \mathrm{D} \cap \mathrm{K}} m_{\mathfrak{s}} G_{\mathfrak{s}, \mathrm{D}}
$$

see Fig. 1. The velocity fluxes $F_{\sigma \mathrm{K}}$ and $F_{\sigma^{*} \mathrm{~K}^{*}}$ are conservative, that is to say $F_{\sigma \mathrm{K}}=-F_{\sigma \mathrm{L}}, \forall \sigma=\mathrm{K} \mid \mathrm{L}$ and $F_{\sigma^{*} \mathrm{~K}^{*}}=-F_{\sigma^{*} \mathrm{~L}^{*}}, \forall \sigma^{*}=\mathrm{K}^{*} \mid \mathrm{L}^{*}$. Next, since $\int_{\mathrm{K}}(\mathbf{u} \cdot \nabla) \mathbf{v}=$ $\sum_{\sigma \subset \partial \mathrm{K}} \int_{\sigma}\left(\mathbf{u} \cdot \mathbf{n}_{\sigma \mathrm{K}}\right) \mathbf{v}$ holds for any $\mathrm{K} \in \mathfrak{M}$, we approximate the convection terms as follows

$$
\int_{\mathrm{K}}(\mathbf{u} \cdot \nabla) \mathbf{v} \rightsquigarrow \sum_{\sigma \subset \partial \mathrm{K}} m_{\sigma} F_{\sigma \mathrm{K}}\left(\frac{\mathbf{v}_{\mathrm{K}}+\mathbf{v}_{\mathrm{L}}}{2}\right),
$$

with a centered discretization for $\mathbf{v}$. For the dual edges the definition is similar.
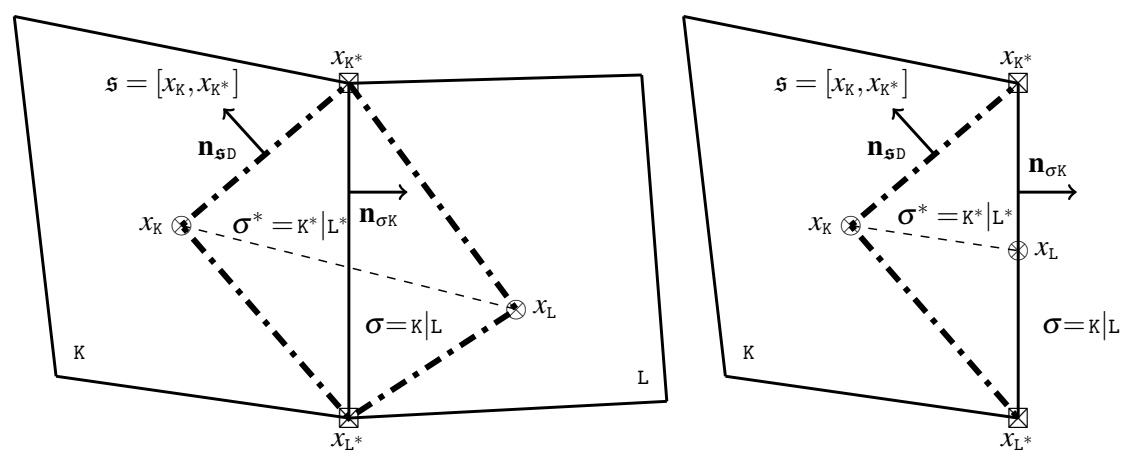

Fig. 1 Left: A diamond $\mathrm{D}=\mathrm{D}_{\sigma, \sigma^{*}}$ with $\sigma \notin \partial \Omega$. Right: A diamond $\mathrm{D}=\mathrm{D}_{\sigma, \sigma^{*}}$ with $\sigma \in \partial \Omega$.

\section{DDFV scheme for the Navier-Stokes equations}

The DDFV scheme under consideration is obtained by an implicit Euler time discretization, except for the nonlinear term, which is linearized by using a semiimplicit approximation. Let $N \in \mathbb{N}^{*}$. We note $\delta t=\frac{T}{N}$ and $t_{n}=n \delta t$ for $n \in\{0, \ldots, N\}$. We look for $\mathbf{u}_{\mathfrak{T}}^{[0, T]}=\left(\mathbf{u}^{n}\right)_{n \in\{0, \ldots N\}} \in\left(\mathbb{E}_{0}\right)^{N+1}$ and $\mathrm{p}_{\mathfrak{D}}^{[0, T]}=\left(\mathrm{p}^{n}\right)_{n \in\{1, \ldots N\}} \in\left(\mathbb{R}^{\mathfrak{D}}\right)^{N+1}$, and the scheme is initialized with $\mathbf{u}^{0}=\mathbb{P}_{c}^{\mathfrak{T}} \mathbf{u}_{0}$ in $\mathbb{E}_{0}$.

To simplify the notations, we denote $\left(\mathbf{u}^{n+1}, \mathrm{p}^{n+1}\right)$ with $\left(\mathbf{u}_{\mathfrak{T}}, \mathrm{p}_{\mathfrak{D}}\right)$ and $\left(\mathbf{u}^{n}, \mathrm{p}^{n}\right)$ with $\left(\overline{\mathbf{u}}_{\mathfrak{T}}, \overline{\mathrm{p}}_{\mathfrak{D}}\right)$ that at each time step are known. Given $\left(\overline{\mathbf{u}}_{\mathfrak{T}}, \overline{\mathrm{p}}_{\mathfrak{D}}\right)$, we look for $\left(\mathbf{u}_{\mathfrak{T}}, \mathrm{p}_{\mathfrak{D}}\right) \in$ $\mathbb{E}_{0} \times \mathbb{R}^{\mathfrak{D}}$ such that: 
FVCAIX, 040, v4 (final): 'Convergence study of a DDFV scheme for the Navier-Stokes...

A DDFV scheme for the Navier-Stokes equations

$$
\left\{\begin{aligned}
m_{\mathrm{K}} \frac{\mathbf{u}_{\mathrm{K}}}{\delta t}+\sum_{\sigma \in \partial \mathrm{K}} m_{\sigma} \mathscr{F} \sigma \mathrm{K} & =m_{\mathrm{K}} \mathbf{f}_{\mathrm{K}}+m_{\mathrm{K}} \frac{\overline{\mathbf{u}}_{\mathrm{K}}}{\delta t} & & \forall \mathrm{K} \in \mathfrak{M} \\
m_{\mathrm{K}^{*}} \frac{\mathbf{u}_{\mathrm{K}^{*}}}{\delta t}+\sum_{\sigma^{*} \in \partial \mathrm{K}^{*}} m_{\sigma^{*}} \mathscr{F}_{\sigma^{*} \mathrm{~K}^{*}} & =m_{\mathrm{K}^{*}} \mathbf{f}_{\mathrm{K}^{*}}+m_{\mathrm{K}^{*}} \frac{\overline{\mathbf{u}}_{\mathrm{K}^{*}}}{\delta t} & & \forall \mathrm{K}^{*} \in \mathfrak{M}^{*} \\
m_{\mathrm{D}} \operatorname{div}^{\mathrm{D}}\left(\mathbf{u}_{\mathfrak{T}}\right) & =0 & & \forall \mathrm{D} \in \mathfrak{D} \\
\sum_{\mathrm{D} \in \mathfrak{D}} m_{\mathrm{D}} \mathrm{p}^{\mathrm{D}} & =0, & &
\end{aligned}\right.
$$

The total fluxes $\mathscr{F}_{\sigma \mathrm{K}}, \mathscr{F}_{\sigma^{*} \mathrm{~K}^{*}}$ read

$$
\begin{aligned}
& m_{\sigma} \mathscr{F}_{\sigma \mathrm{K}}=-m_{\sigma} \sigma^{\mathrm{D}}\left(\mathbf{u}_{\mathfrak{T}}, \mathrm{p}_{\mathfrak{D}}\right) \mathbf{n}_{\sigma \mathrm{K}}+\left[m_{\sigma} F_{\sigma \mathrm{K}}\left(\frac{\mathbf{u}_{\mathrm{K}}+\mathbf{u}_{\mathrm{L}}}{2}\right)+\frac{m_{\sigma}^{2}}{2 \operatorname{Re} m_{\mathrm{D}}} B_{\sigma \mathrm{K}}\left(\mathbf{u}_{\mathrm{K}}-\mathbf{u}_{\mathrm{L}}\right)\right] \\
& m_{\sigma^{*}} \mathscr{F}_{\sigma^{*} \mathrm{~K}^{*}}=-m_{\sigma^{*}} \sigma^{\mathrm{D}}\left(\mathbf{u}_{\mathfrak{T}}, \mathrm{p}_{\mathfrak{D}}\right) \mathbf{n}_{\sigma^{*} \mathrm{~K}^{*}} \\
& +\left[m_{\sigma^{*}} F_{\sigma^{*} \mathrm{~K}^{*}}\left(\frac{\mathbf{u}_{\mathrm{K}^{*}}+\mathbf{u}_{\mathrm{L}^{*}}}{2}\right)+\frac{m_{\sigma^{*}}^{2}}{2 \operatorname{Re} m_{\mathrm{D}}} B_{\sigma^{*} \mathrm{~K}^{*}}\left(\mathbf{u}_{\mathrm{K}^{*}}-\mathbf{u}_{\mathrm{L}^{*}}\right)\right] .
\end{aligned}
$$

They are the sum of a "diffusion" term, discretized by means of the DDFV operators defined in Sec. 2, and a "convection" term, approximated through general $B$ schemes, as in $[2,8]$. It means that the latter are written as a centered discretization plus a diffusive perturbation, which depends on a certain function $B$. The definition of the velocity fluxes $F_{\sigma K}, F_{\sigma^{*} K^{*}}$ comes from the literature and it can be found in Sec. 2; they are computed with the velocity of the previous time step. We now need to define the matrices $B_{\sigma \mathrm{K}}, B_{\sigma^{*} \mathrm{~K}^{*}}$.

\section{Definition of the diffusive perturbations to the convection fluxes.}

Our study is motivated by domain decomposition purposes: the domain $\Omega$ is seen as the union of two subdomains that share a common interface $\Gamma$. A specific definition of the total fluxes is required on the interface, as a trace of the iteration process [7]. The diamonds of $\Omega$ which cross the interface $\Gamma$ are split into two boundary diamonds on the subdomains; they share the primal edge $\sigma$, which lies on the interface $\Gamma$, while the dual edge $\sigma^{*}$ is divided into $\sigma^{*} \cap \mathrm{K}$ and $\sigma^{*} \cap \mathrm{L}$, see Fig. 1 . The convergence of the Schwarz algorithm amounts to re-glue the two pieces of such diamonds. This entails the following properties on the total fluxes

$$
\begin{aligned}
m_{\sigma} \mathscr{F}_{\sigma \mathrm{K}} & =-m_{\sigma} \mathscr{F}_{\sigma \mathrm{L}} \\
m_{\sigma^{*}} \mathscr{F}_{\sigma^{*} \mathrm{~K}^{*}} & =m_{\sigma^{*} \cap \mathrm{K}} \mathscr{F}_{\sigma^{*} \cap \mathrm{K}, \mathrm{K}^{*}}+m_{\sigma^{*} \cap \mathrm{L}} \mathscr{F}_{\sigma^{*} \cap \mathrm{L}, \mathrm{K}^{*}}
\end{aligned}
$$

which are not naturally satisfied. Relations (2) lead to algebraic constraints, which, in turn, modify the definition of the coefficients $B_{\sigma K}, B_{\sigma^{*} K^{*}}$ on the interface. In particular it leads to work with matrix-valued $B_{\sigma \mathrm{K}}, B_{\sigma^{*} \mathrm{~K}^{*}}$. Therefore, for the primal mesh, we have 


$$
B_{\sigma \mathrm{K}}:= \begin{cases}B\left(\frac{2 \operatorname{Re} m_{\mathrm{D}}}{m_{\sigma}} F_{\sigma \mathrm{K}}\right) \mathrm{Id} & \forall \mathrm{D}_{\sigma, \sigma^{*}} \in \mathfrak{D} \backslash \mathfrak{D}^{\Gamma} \\ \frac{2 \operatorname{Re} m_{\mathrm{D}}}{m_{\sigma}^{2}}\left(A_{\mathrm{K}} A_{\mathrm{L}}+\left(\frac{1}{2} m_{\sigma} F_{\sigma \mathrm{K}}\right)^{2} \mathrm{Id}\right) A^{-1}-P & \forall \mathrm{D}_{\sigma, \sigma^{*}} \in \mathfrak{D}^{\Gamma}\end{cases}
$$

with $P=\mathrm{Id}+\mathbf{n}_{\sigma \mathrm{K}} \otimes \mathbf{n}_{\sigma \mathrm{K}}$ for $\sigma=\mathrm{K} \mid \mathrm{L}$ and

$$
A_{\mathrm{K}}:=\frac{m_{\sigma}^{2}}{2 \operatorname{Re} m_{\mathrm{D} \cap \mathrm{K}}}\left(P+B\left(\frac{2 \operatorname{Re} m_{\mathrm{D} \cap \mathrm{K}}}{m_{\sigma}} F_{\sigma \mathrm{K}}\right) \mathrm{Id}\right), \quad A:=A_{\mathrm{K}}+A_{\mathrm{L}} .
$$

For the dual mesh, we have

$B_{\sigma^{*} \mathrm{~K}^{*}}= \begin{cases}B\left(\frac{2 \mathrm{Re} m_{\mathrm{D}}}{m_{\sigma^{*}}} F_{\sigma^{*} \mathrm{~K}^{*}}\right) \mathrm{Id}, & \forall \mathrm{D}_{\sigma, \sigma^{*}} \in \mathfrak{D} \backslash \mathfrak{D}^{\Gamma} \\ \frac{m_{\sigma^{*} \cap \mathrm{K}}}{m_{\sigma^{*}}} B\left(\frac{2 \operatorname{Re} m_{\mathrm{D} \cap \mathrm{K}}}{m_{\sigma^{*} \cap \mathrm{K}}} F_{\sigma^{*} \cap \mathrm{K}}\right) \mathrm{Id}+\frac{m_{\sigma^{*} \cap \mathrm{L}}}{m_{\sigma^{*}}} B\left(\frac{2 \operatorname{Re} m_{\mathrm{D} \cap \mathrm{L}}}{m_{\sigma^{*} \cap \mathrm{L}}} F_{\sigma^{*} \cap \mathrm{L}}\right) & \mathrm{Id}, \quad \forall \mathrm{D}_{\sigma, \sigma^{*}} \in \mathfrak{D}^{\Gamma}\end{cases}$

where, for $\mathrm{D}_{\sigma, \sigma^{*}} \in \mathfrak{D}^{\Gamma}$, we set $m_{\sigma^{*} \cap \mathrm{K}} F_{\sigma^{*} \cap \mathrm{K}}=-m_{\mathfrak{s}} G_{\mathfrak{s}, \mathrm{D}}-\frac{1}{2} \sum_{\sigma \subset \mathrm{K}^{*} \cap \Gamma} m_{\sigma \cap K^{*}} \overline{\mathbf{u}}_{\mathrm{K}^{*}} \cdot \mathbf{n}_{\sigma \mathrm{K}}$.

Therefore the details of the fluxes depend on the function $B$ which appears in these definitions. On the interior diamonds $\mathfrak{D} \backslash \mathfrak{D}^{\Gamma}$, for both primal and dual meshes, standard choices are $B(s)=0$ which leads to the centered scheme, or $B(s)=\frac{1}{2}|s|$, which corresponds to the upwind scheme. We refer the reader to [11] for the analysis of the DDFV scheme for (1) with the upwind scheme on the entire domain $\Omega$. This result generalizes as follows.

Theorem 1. Let $\mathfrak{T}$ be a mesh that satisfies inf-sup stability condition and let $B$ be an even Lipschitz continuous function such that $B(s) \geq 0, \forall \mathfrak{s} \in \mathbb{R}$. Then, the problem ( $\widetilde{P})$ is well-posed.

The hypothesis of inf-sup stability ([1]) on the mesh can be dropped by stabilizing the incompressibility constraint. For the proof, we refer the reader to [7].

\section{Numerical results}

In this Section, the scheme $(\widetilde{\mathscr{P}})$ is validated by some numerical experiments. The computational domain is $\Omega=[-1,1] \times[0,1]$ and the interface $\Gamma$ is placed at $x=0$. For the tests, we give the expression of the exact solution $(\mathbf{u}, p)$, from which we deduce the source term $\mathbf{f}$. We compare the $L^{2}$-norm of the error (difference between a centered projection of the exact solution and the approximated solution obtained with DDFV scheme) for the velocity (denoted Ervel), the velocity gradient (Ergradvel) and the pressure (Erpre). The error estimates are discussed by working with a family of meshes (see Fig. 2), obtained by refining successively and uniformly the original mesh. The sub-index in the name of the mesh denotes the level of refinement, i.e. Mesh ${ }_{1}^{k}$ represents the coarse mesh of a family of refined meshes $\left(\mathrm{Mesh}_{m}^{k}\right)_{m}$. More precisely, $\operatorname{Mesh}_{m}^{k}$ is obtained by dividing by two all the edges of $\operatorname{Mesh}_{m-1}^{k}$. The meshes in those examples are non conformal. 
We consider the following exact solutions to (1):

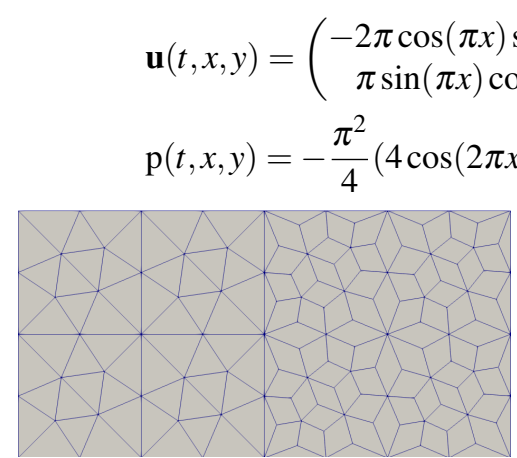

(a) $\operatorname{Mesh}_{1}^{1}$

Fig. 2 Coarse level of refinement of the meshes on $\Omega$

The final time is $T=0.3$ and we fix $\delta t=1.5 \times 10^{-3}, \eta=\operatorname{Re}=1$, and $B(s)=\frac{1}{2}|s|$. In Tables 1 and 2, we observe convergence of order 1 for the $L^{2}$ norm of the velocity, the $H^{1}$ norm of the velocity and for the $L^{2}$ norm of the pressure. Those results are comparable to the ones presented in [11]. This underlines that the presence of the interface $\Gamma$ and the modified fluxes that appear in (3),(4) do not influence the convergence results. The solution of $(\widetilde{\mathscr{P}})$ is a good approximation of the solution of (1).

Table 1 Test (5) on $\operatorname{Mesh}_{m}^{1}, m=1, \ldots 5$.

\begin{tabular}{|c|c|c|c|c|c|c|c|}
\hline Mesh & JbCell & I Ervel & Ratio & Ergradvel & Ratio & Erpre & Ratio \\
\hline 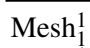 & & & & & & & \\
\hline & & & & & & & \\
\hline & & & & & & & \\
\hline $\operatorname{Mesh}_{4}^{1}$ & 4947 & & 0.97 & & 1.00 & & 1.44 \\
\hline $\operatorname{Mesh}_{5}^{1}$ & 195716 & $6.802 \mathrm{E}-004$ & 1.13 & $4.594 \mathrm{E}-003$ & 1.01 & $2.772 \mathrm{E}-002$ & 1.30 \\
\hline
\end{tabular}

Table 2 Test (5) on $\operatorname{Mesh}_{m}^{2}, m=1, \ldots 5$.

\begin{tabular}{|c|c|c|c|c|c|c|c|}
\hline Mesh & NbCell & I Ervel & Ratio & Ergradvel & Ratio & Erpre & Rati \\
\hline & & & 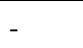 & & . & & \\
\hline & 33 & 1.9 & 2.10 & & & 2 & 34 \\
\hline & & & 2 & & 1 & 0. & 1.58 \\
\hline & 4902 & & 1.37 & & 1.09 & 0.2 & 1.65 \\
\hline $\operatorname{esh}_{5}^{2}$ & 193284 & 7.725E-004 & 1.23 & $5.460 \mathrm{E}-003$ & 1.05 & 7.083E-002 & 1.59 \\
\hline
\end{tabular}




\section{References}

[1] F. Boyer, S. Krell, and F. Nabet, Inf-Sup stability of the Discrete Duality Finite Volume method for the 2D Stokes problem, Math. Comput. 84 (2015), 27052742.

[2] C. Chainais-Hillairet and J. Droniou, Finite volume schemes for non-coercive elliptic problems with Neumann boundary conditions, IMA J. Numer. Anal. 31 (2011), 61-85.

[3] Y. Coudière, J.-P. Vila, and P. Villedieu, Convergence rate of a finite volume scheme for a two dimensional convection-diffusion problem, ESAIM: Math. Mod. Numer. Anal. 33 (1999), no. 3, 493-516.

[4] S. Delcourte and P. Omnes, A Discrete Duality Finite Volume discretization of the vorticity-velocity-pressure formulation of the $2 D$ Stokes problem on almost arbitrary two-dimensional grids, Numer. Methods PDEs (2015), 1-30.

[5] K. Domelevo and P. Omnes, A finite volume method for the Laplace equation on almost arbitrary two-dimensional grids, ESAIM: Math. Mod. Numer. Anal. 39 (2005), no. 6, 1203-1249.

[6] T. Goudon, S. Krell, and G. Lissoni, DDFV method for Navier-Stokes problem with outflow boundary conditions, Numer. Math. 142 (2019), no. 1, 55-102.

[7] _ Non-overlapping Schwarz algorithms for the incompressible NavierStokes equations with DDFV discretizations, Tech. report, Univ. Côte d'Azur, Inria, CNRS, LJAD, 2019, Available at https://hal.archives-ouvertes.fr/hal02448007.

[8] L. Halpern and F. Hubert, A finite volume Ventcell-Schwarz algorithm for advection-diffusion equations, SIAM J. Numer. Anal. 52 (2014), no. 3, 12691291.

[9] F. Hermeline, A finite volume method for the approximation of diffusion operators on distorted meshes, J. Comput. Phys. 160 (2000), no. 2, 481-499.

[10] S. Krell, Stabilized DDFV schemes for Stokes problem with variable viscosity on general 2D meshes, Numer. Methods PDEs 27 (2011), no. 6, 1666-1706.

[11] Stabilized DDFV schemes for the incompressible Navier-Stokes equations, Finite Volumes for Complex Applications VI, Problems \& Perspectives, 2011, pp. 605-612.

[12] P. L. Lions, On the Schwarz alternating method. III. A variant for nonoverlapping subdomains, Third International Symposium on Domain Decomposition Methods for Partial Differential Equations, SIAM, Philadelphia, PA, 1990, pp. 202-223.

[13] H.A. Schwarz, Über einen grenzübergang durch alternierendes verfahren, Vierteljahrsschrift der Naturforschenden Gesellschaft in Zurich 15 (1870), 272-286.

The paper is in final form and no similar paper has been or is being submitted elsewhere. 